\title{
High Energy Gamma Rays from $\mathbf{p}+\mathbf{X}$, $\mathrm{X}=\mathrm{Cu}, \mathrm{Ag}, \mathrm{Au}$ at $34 \mathrm{MeV}$
}

S.J. Luke, F.S. Dietrich, M.B. Chadwick, C.A. Gossett, M.S. Kaplan, B.T. McLain, R. Vandenbosch, M. KicinskaHabior

This article was submitted to

$10^{\text {th }}$ International Symposium on Capture Gamma-Ray Spectroscopy and Related Topics, Santa Fe, NM, August 30 September 3, 1999

\section{March 7, 2000}

U.S. Department of Energy

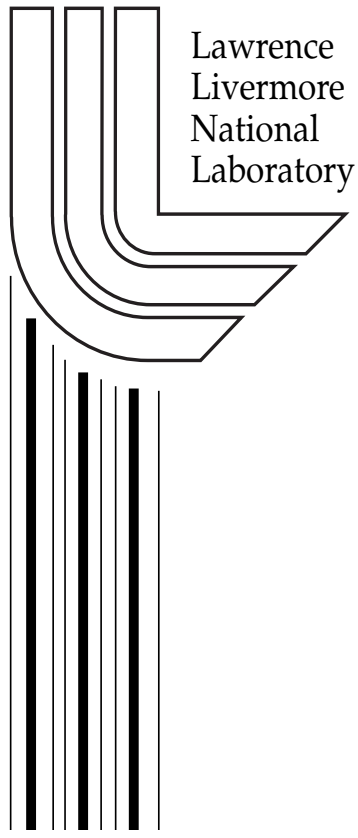




\section{DISCLAIMER}

This document was prepared as an account of work sponsored by an agency of the United States Government. Neither the United States Government nor the University of California nor any of their employees, makes any warranty, express or implied, or assumes any legal liability or responsibility for the accuracy, completeness, or usefulness of any information, apparatus, product, or process disclosed, or represents that its use would not infringe privately owned rights. Reference herein to any specific commercial product, process, or service by trade name, trademark, manufacturer, or otherwise, does not necessarily constitute or imply its endorsement, recommendation, or favoring by the United States Government or the University of California. The views and opinions of authors expressed herein do not necessarily state or reflect those of the United States Government or the University of California, and shall not be used for advertising or product endorsement purposes.

This is a preprint of a paper intended for publication in a journal or proceedings. Since changes may be made before publication, this preprint is made available with the understanding that it will not be cited or reproduced without the permission of the author.

This report has been reproduced

directly from the best available copy.

Available to DOE and DOE contractors from the

Office of Scientific and Technical Information

P.O. Box 62, Oak Ridge, TN 37831

Prices available from (423) 576-8401

http://apollo.osti.gov/bridge/

Available to the public from the

National Technical Information Service

U.S. Department of Commerce

5285 Port Royal Rd.,

Springfield, VA 22161

http://www.ntis.gov/

OR

Lawrence Livermore National Laboratory

Technical Information Department's Digital Library

http://www.llnl.gov/tid/Library.html 


\title{
High Energy Gamma Rays from $p+X$, $\mathrm{X}=\mathrm{Cu}, \mathrm{Ag}, \mathrm{Au}$ at $34 \mathrm{MeV}$
}

\author{
S.J. Luke, F.S. Dietrich, M.B. Chadwick ${ }^{1}$ \\ Lawrence Livermore National Laboratory, Livermore, CA 94550 \\ C.A. Gossett, M.S. Kaplan, B.T. McLain, R. Vandenbosch \\ Nuclear Physics Laboratory, University of Washington, Seattle, WA 98195 \\ M. Kicinska-Habior \\ Institute of Experimental Physics, University of Warsaw, Poland
}

\begin{abstract}
In this paper we present results from the measurement of the gamma ray yield in the reaction of $34-\mathrm{MeV}$ protons on $\mathrm{Cu}, \mathrm{Ag}$ and $\mathrm{Au}$. The protons were produced by the University of Washington superconducting linac. The gamma rays were measured using a large $\mathrm{NaI}$ and two large $\mathrm{BaF}_{2}$ detectors. Angular distributions were obtained for each of the three targets. Data for the $\mathrm{Cu}$ and $\mathrm{Ag}$ target were taken at six lab angles between 35 and 135 degrees, while data were taken at eight lab angles between 35 and 135 degrees for the Au target. The data were compared to several models. These included Hauser-Feshbach and direct-semidirect (DSD) calculations. We also compared the measurements to proton-nucleus bremsstrahlung calculations. The bremsstrahlung calculations greatly underpredicted the cross section and produced an angular distribution which was too flat. The Hauser-Feshbach calculations reproduced the yield of the softer portion of the spectrum reasonably well for all three targets. The DSD calculations reproduced the yield and angular distributions quite well for energies above about $20 \mathrm{MeV}$. However, the yields were underpredicted in the 15-18 MeV region, which suggests that multistep mechanisms may be needed for this target.
\end{abstract}

\section{INTRODUCTION}

This work had two essential motivations. The first was to complement a series of heavy ion experiments $[1,2]$, in order to understand the production mechanism of high energy $\gamma$ rays at intermediate energies. The argument for this motivation is as follows. If the production mechanism for high energy $\gamma$ rays in heavy-ion

1) Present address: Los Alamos National Laboratory. 
experiments is nucleon-nucleon bremsstrahlung, then the $(\mathrm{p}, \gamma)$ reaction producing $\gamma$ rays above the GDR (Giant Dipole Resonance) should serve as a check of such bremsstrahlung mechanisms [3]. If the mechanism is uncontaminated by competing mechanisms then $(\mathrm{p}, \gamma)$ reactions could serve as a calibration of the bremsstrahlung mechanism in other reactions. The unique feature of proton induced reactions is the fact that in these reactions the system accesses a vastly different kinematic region than heavy ion collisions, especially near the kinematic limit for the production of the $\gamma$. What we discovered was that there was significant contribution from other reaction mechanisms, particularly the direct-semidirect (DSD) mechanism discussed below.

The second motivating factor was the attempt to extend our understanding of the DSD reaction mechanism for capture to unbound final states observed in the ${ }^{89} \mathrm{Y}(\vec{p}, \gamma)$ reaction with $19.6-\mathrm{MeV}$ protons. A DSD model with direct $\mathrm{E} 1, \mathrm{E} 2$, and $\mathrm{E} 3$ and semidirect E1 radiation was developed to reproduce the data for an experiment performed at TUNL [4]. The model has worked quite well in explaining the data, both at $19.6 \mathrm{MeV}$ and the higher energies in the present work. However, the DSD model, in combination with a Hauser-Feshbach calculation, underpredicts the yields in the range of $15-18 \mathrm{MeV}$. This result suggests that additional multistep processes may be necessary.

\section{EXPERIMENTAL DETAILS}

The measurements were performed at the University of Washington Nuclear Physics Laboratory. The proton beam was delivered by the Tandem Van de Graff/Superconducting Linac combination. The proton beam bombarded $\mathrm{Cu}, \mathrm{Ag}$ and $\mathrm{Au}$ targets. These targets were chosen to extend the systematics of photon production in these systems from the work of the Grenoble group at 72 and $168 \mathrm{MeV}[7,8]$, respectively. The targets were relatively thick (approximately 5 $\mathrm{mg} / \mathrm{cm}^{2}$ ) freestanding metal targets. The beam was bunched and chopped so that time-of-flight could be utilized to separate the high energy $\gamma$ rays from high energy neutrons. Absolute normalization was accomplished by means of a Faraday cup. Angular distribution data were taken at eight angles for the gold target and six angles each for the silver and copper targets.

Two types of detectors were used to detect the high energy $\gamma$ rays. These were a large $(25.4 \times 38.1 \mathrm{~cm}) \mathrm{NaI}$ spectrometer and two large volume $(14.5 \times 17.0$ cm) $\mathrm{BaF}_{2}$ spectrometers. The construction of these detectors was very similar. The central crystal was surrounded by an active anticoincidence shield, passive lead shielding, and neutron shielding between the crystal and the target. The anticoincidence shield served two purposes: first, it vetoed cosmic rays (a large source of background in this type of experiment) and second, it allowed for the veto of any events which might partially leak out of the detector, thereby improving the line shape characteristics of the detector. The solid angles for the $\mathrm{NaI}, \mathrm{BaF}_{2} \# 1$ and $\mathrm{BaF}_{2} \# 2$ were 78.5, 57.5 and 51.76 msr, respectively. 
The detectors were energy calibrated before and after the main data taking, using the reaction ${ }^{11} \mathrm{~B}\left(\mathrm{p}, \gamma_{0,1}\right)$ at proton energies of 7.25 and $14.3 \mathrm{MeV}$. These reactions yielded $\gamma$ rays with energies of $18.2,22.6,24.7$, and $29.1 \mathrm{MeV}$. In addition, the calibration was checked periodically during the data acquisition process by the use of ${ }^{12} \mathrm{C}\left(\mathrm{p}, \gamma_{0}\right)$ at $34 \mathrm{MeV}$, which produced $\gamma$ rays with energies 33.3 and $28.9 \mathrm{MeV}$ from the $\gamma_{0}$ and the $\gamma_{1}$ decays, respectively. These frequent checks assured that the gain of the detectors was stable throughout the course of the measurements.

The absolute efficiency-solid angle product, $\epsilon \mathrm{d} \Omega$, of the detectors was determined by measuring the absolute resonance yield for the reaction ${ }^{12} \mathrm{C}\left(\mathrm{p}, \gamma_{0}\right)$ at $14.25 \mathrm{MeV}$, and comparing this value to the measurement of Marrs et al. [9]. This measurement allowed the determination of the absolute efficiency at a $\gamma$ ray energy of $15.1 \mathrm{MeV}$. In order to use the measurements of Marrs et al. as the basis for efficiency calibration at other energies, Monte Carlo simulations were carried out. These simulations showed that the total efficiency of the detectors was nearly constant with energy, while the "accept" efficiency, defined by those events not in coincidence with the plastic anticoincidence shield, falls off exponentially as function of energy above 15.1 MeV. The exponential slope of the "accept" efficiency was obtained from the measurement of the accept-to-total ratio for the $\gamma$ rays from ${ }^{11} \mathrm{~B}\left(\mathrm{p}, \gamma_{0,1}\right)$ at various incident proton energies.

As mentioned above, events from cosmic rays constituted the major source of the experimental background in these measurements. Approximately 98-99\% cosmic rejection was obtained in the region of interest by using the active anticoincidence shield. Additional cosmic ray rejection was obtained by using a pulsed beam. Because of the narrow time window $(2 \mathrm{~ns})$ of the $\gamma$ rays and the time between beam bursts of $240 \mathrm{~ns}$, an additional two orders of magnitude in rejection of cosmic rays was achieved. Random cosmic ray background was subtracted in the offline analysis. Two additional sources of background, high energy neutrons and $\gamma$ rays from beam-related events other than the beam striking the target,were rejected by time-of-flight (TOF).

The data were acquired using an MBD-11 computer which read the ADC's and QDC's. The MBD-11 handed the data to a VAXstation 3100 which was responsible for histogramming the data and writing the data directly to the hard disk, through the program XSYS. The master gate of the electronics was an "OR" between the $\mathrm{NaI}$ and the two $\mathrm{BaF}_{2}$ detectors. Each of the detectors had an "accept" and a "reject" route based upon the absence or presence of a coincidence with the plastic anticoincidence shield, respectively. The TOF spectra were gated with the same "accept" and "reject" routes as the energy signals. All data were placed in twodimensional spectra (energy versus TOF) for offline analysis. The average data acquisition dead time was $2 \%$.

The data were analyzed in a two-dimensional manner using the energy versus time-of-flight spectra. Narrow time gates were determined for the $\gamma$ ray peaks and also for the random background. The data were then replayed and put into one-dimensional energy spectra. The spectra for the random background were then subtracted from the spectra from the prompt $\gamma$ rays, yielding the raw energy 


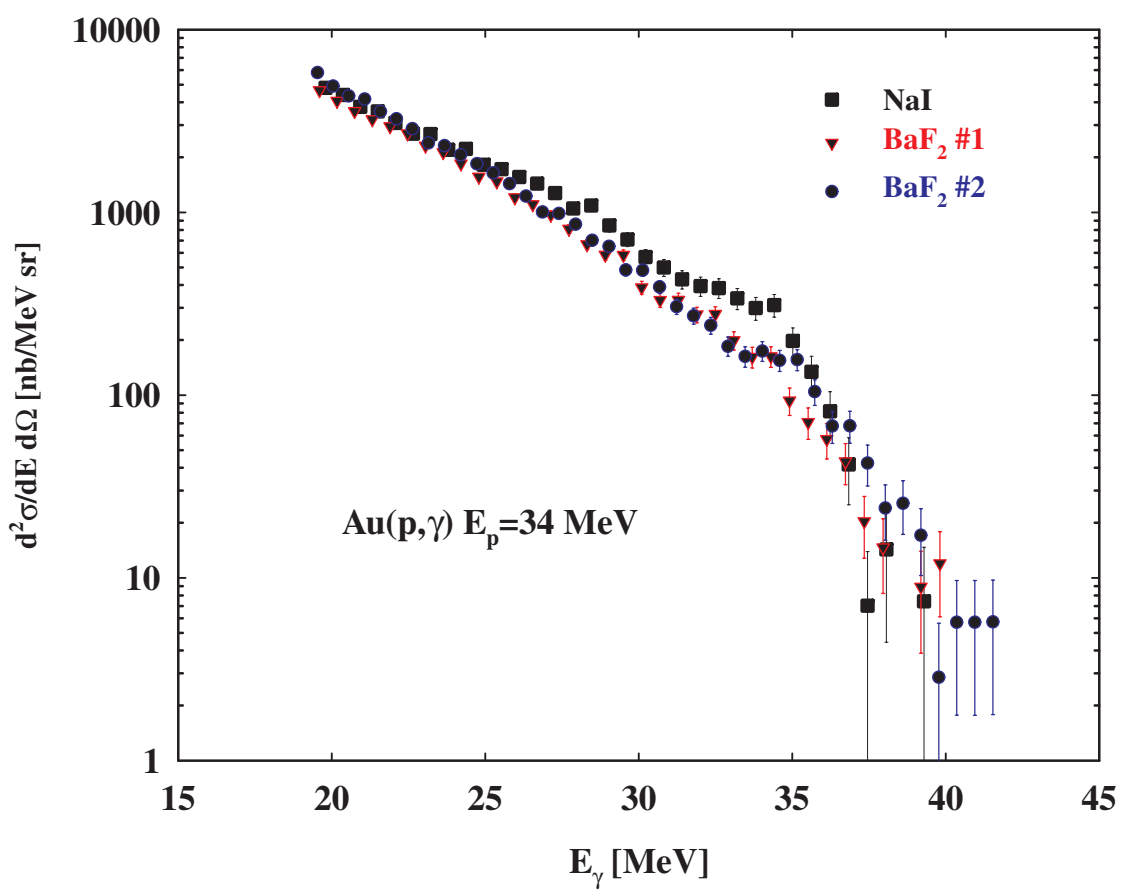

FIGURE 1. Gamma yield at 90 degrees with the Au target and all three detectors.

spectra. These raw spectra were converted to doubly differential cross sections by using the values for the measured efficiency, the target thickness, and the total integrated charge in the Faraday cup.

An example of the data, for the $\mathrm{Au}$ target, is shown in figure 1. This figure shows that the measurement is reasonably consistent for each of the detectors. The small differences in the yields are the result of the differences in the response functions of the three detectors; the spectra have not been corrected for these differences in efficiency. The spectra are relatively featureless. An important feature of the observed yield is that the kinematic limit (i.e. upper energy limit) for the production of the $\gamma$ rays is accessed. This corresponds roughly to the region just above the "knee" at the upper end of the spectrum. We will display the angular distribution in section III.

\section{CALCULATIONS}

Two types of calculations were performed, and before comparing with the data the calculations were convoluted with the response functions of the detectors. The first calculation was a modification of the nucleon-exchange transport model used for heavy ion interactions [3]. This model assumes that the $\gamma$ rays are produced by nucleon-nucleon bremsstrahlung, specifically proton-neutron bremsstrahlung. The bremsstrahlung was calculated both classically and quantum mechanically. The 
quantum mechanical calculations were determined by fits to the calculation of Cassing et al. [5]; the procedure for this is found in ref. [1]. The calculations of the photon yield in this model were carried out using both diffuse and sharp momentum distributions for the target nucleons. The results of these calculations are shown in figure 2 for the Au case. As is clearly seen in this figure, these calculation do not reproduce the absolute yield or the shape of the angular distributions.

The second type of calculation is based on a direct-semidirect model which originally incorporated direct E1, E2, E3 and semidirect E1 radiation to calculate capture to unbound final states [4]. This DSD model is supplemented by a HauserFeshbach calculation, which is the predominant source of gammas below approximately $15 \mathrm{MeV}$. The combined model worked well at $19.6 \mathrm{MeV}$ incident energy for ${ }^{89} \mathrm{Y}(\vec{p}, \gamma)$ in reproducing the yield as well as the angular distributions and analyzing powers [4]. The present calculations were undertaken to ascertain whether the model works well also at higher energies. For the new calculations, convectivecurrent direct M1 and M2 radiations were added. These extra multipolarities were shown to have a significant effect on the angular distributions, particularly at forward angles, although the effect on the angle-integrated cross section was small. In
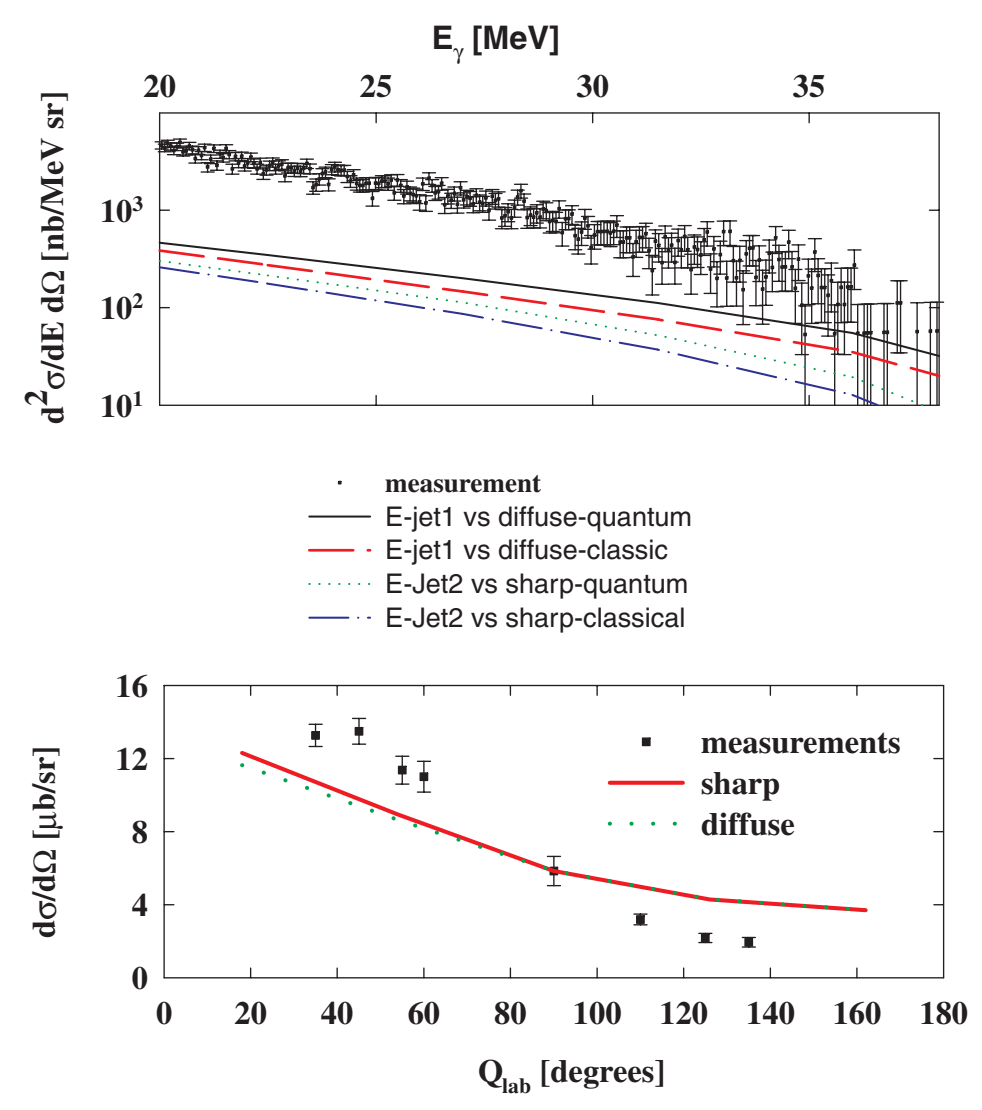

FIGURE 2. Experimental results for the Au target compared to bremsstrahlung calculations. 
addition to adding the magnetic radiations, optical potentials and GDR parameters appropriate to the incident energy and target nuclei were chosen; otherwise, the calculations were carried out as described in ref. [4]. The results of these calculations are shown in figures 3 and 4 . As is seen in figure 4 the calculations reproduce the angular distribution very well, both in shape and magnitude. These angular distributions are for the portion of the energy spectrum integrated from 25 to $33 \mathrm{MeV}$. The calculation of the spectral shape in the region above 16-18 MeV where the DSD mechanism dominates is quite good as well, as shown in figure 3 . However, the model (when added to the Hauser-Feshbach contribution, also shown in the figure), does not fully reproduce the yield of the $\gamma$ rays in the $15-18 \mathrm{MeV}$ region. This might suggest that there is an additional mechanism present, which may be a multistep mechanism. This deficiency was not seen in the experiment at 19.6 MeV.

\section{CONCLUSIONS AND ACKNOWLEDGMENTS}

In this paper we have presented data for measurement of $\gamma$ rays from three targets for incident protons at $34 \mathrm{MeV}$. The data include both energy spectra and angular distributions. Nucleon-nucleon bremsstrahlung cannot explain either the total yield or the angular distributions. However, both can be well explained by a sum of Hauser-Feshbach and direct-semidirect models, except for a deficiency in the neighborhood of $15-18 \mathrm{MeV}$.

The authors wish to thank Dr. Kurt Snover for his illuminating discussions and helpful comments. This work was carried out under the auspices of the U. S. Depart-

ment of Energy under Grant No. DE-FG06-90ER40537 (University of Washington) and Contract No. W-7405-Eng-48 (Lawrence Livermore National Laboratory).

\section{REFERENCES}

1. S. J. Luke, Ph.D. dissertation, University of Washington, 1992.

2. S. J. Luke, R. Vandenbosch, W. Benenson, J. Clayton, K. Joh, D. Krofcheck, T. K. Murakami and J. Stevenson, Phys. Rev. C 47, 1121(1993).

3. S. J. Luke, R. Vandenbosch and J. Randrup, Phys. Rev. C 48, 857(1993).

4. W. E. Parker, F. S. Dietrich, S. J. Luke, K. E. Sale, J. E. Kammeraad, M. B. Chadwick, L. H. Kramer, R. M. Chasteler, M. A. Godwin, G. J. Schmid, H. R. Weller and A. K. Kerman, Phys. Rev. C 52, 252(1995).

5. W. Cassing, V. Metag, U. Metag, Phys. Reports 188, 363(1990).

6. H. Nifenecker and J. Pinston, Ann. Rev. Nucl. Part. Sci. 23, 113(1990).

7. M. Kwato Njock et al., Phys. Lett. 207B, 269(1988).

8. J. Pinston et al., Phys. Lett. 218B, 128(1989).

9. R. E. Marrs, E. A. Adelberger and K. A. Snover, Phys. Rev. C 16, 61(1977).

10. J. Clayton et al., Nucl. Inst. and Meth. A305, 116(1991). 


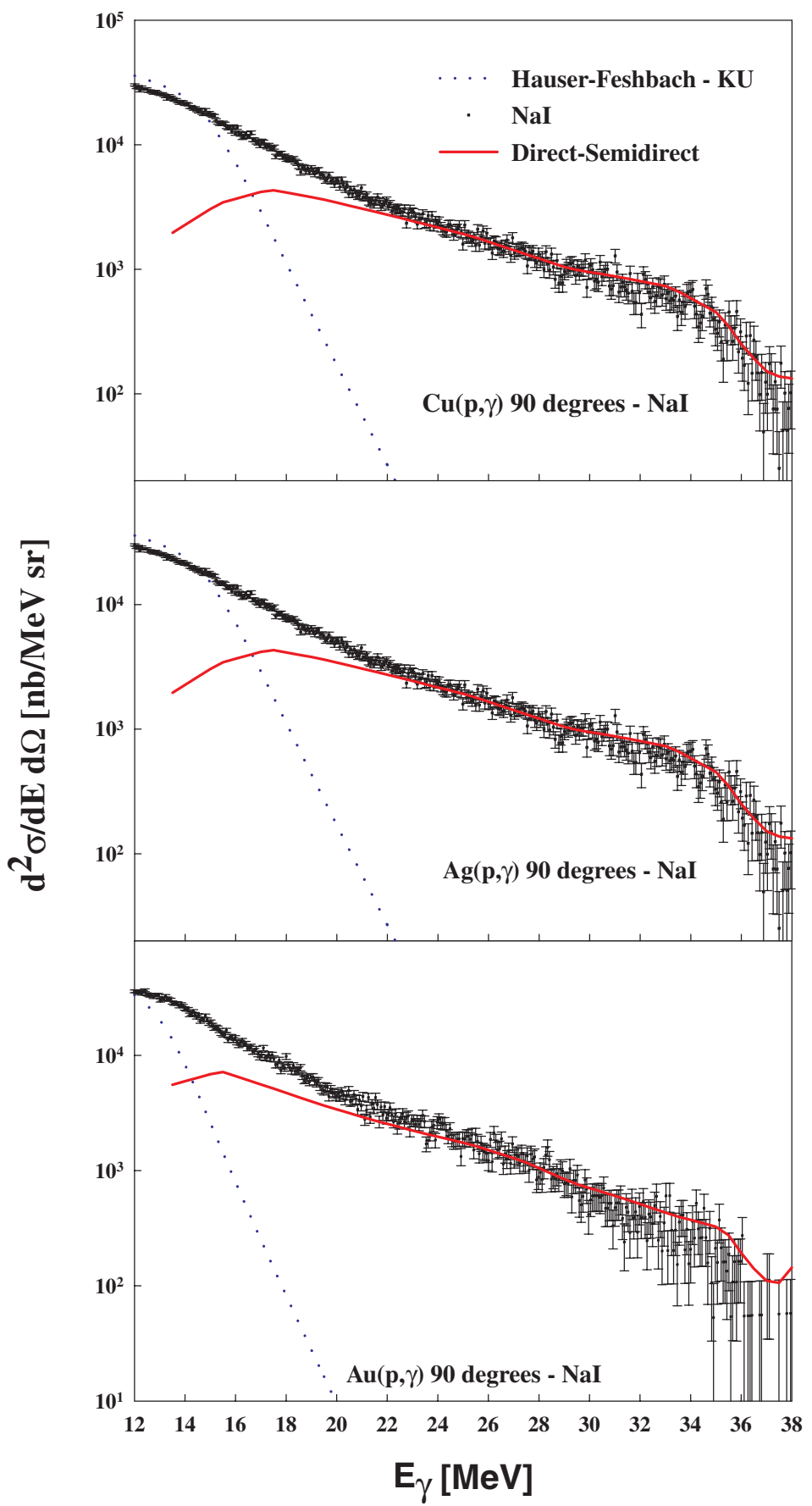

FIGURE 3. Experimental yields at 90 degrees for all three targets compared to direct-semidirect calculations (solid line) and Hauser-Feshbach calculations (dotted line). 


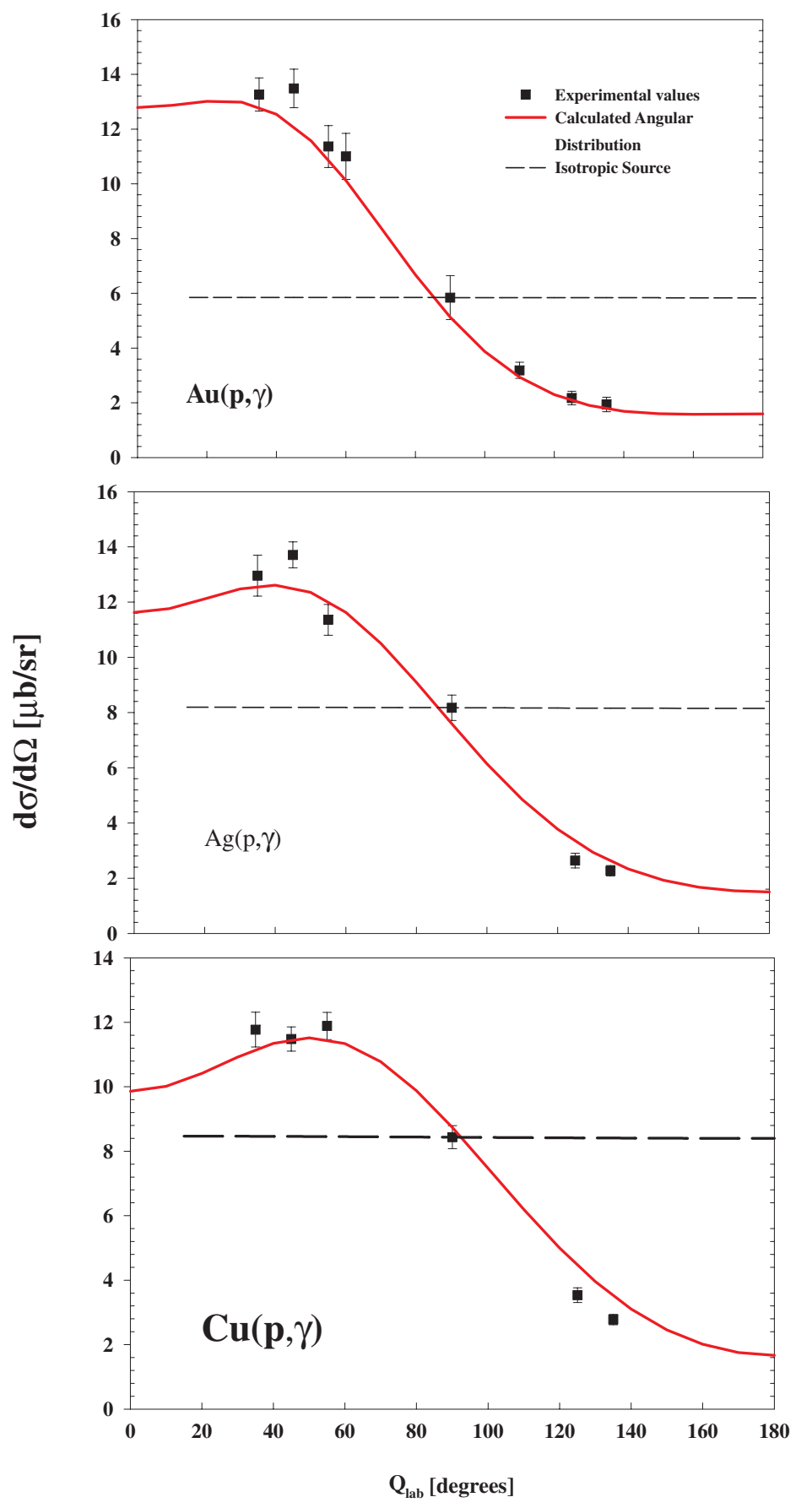

FIGURE 4. Experimental angular distributions for all three targets compared to direct-semidirect calculations (solid lines), and emission from a moving source moving at the velocity of the compound nucleus (dashed lines). The angular distributions are for the portion of the energy spectrum integrated from 25 to $33 \mathrm{MeV}$. 